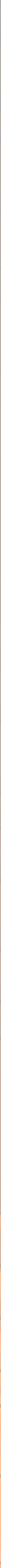




\section{Nef cones of Hilbert schemes of points on surfaces}

\section{Barbara Bolognese, Jack Huizenga, Yinbang Lin, Eric Riedl, Benjamin Schmidt, Matthew Woolf and Xiaolei Zhao}

Let $X$ be a smooth projective surface of irregularity 0 . The Hilbert scheme $X^{[n]}$ of $n$ points on $X$ parametrizes zero-dimensional subschemes of $X$ of length $n$. We discuss general methods for studying the cone of ample divisors on $X^{[n]}$. We then use these techniques to compute the cone of ample divisors on $X^{[n]}$ for several surfaces where the cone was previously unknown. Our examples include families of surfaces of general type and del Pezzo surfaces of degree 1. The methods rely on Bridgeland stability and the positivity lemma of Bayer and Macrì.

A correction was posted on 16 June 2018 in an online supplement.

1. Introduction

2. Preliminaries

910

3. Gieseker walls and the nef cone

915

4. Picard rank 1 examples

922

5. Del Pezzo surfaces of degree $1 \quad 925$

Acknowledgements $\quad 928$

$\begin{array}{ll}\text { References } & 928\end{array}$

\section{Introduction}

If $X$ is a projective variety, the cone $\operatorname{Amp}(X) \subset N^{1}(X)$ of ample divisors controls the various projective embeddings of $X$. It is one of the most important invariants of $X$, and carries detailed information about the geometry of $X$. Its closure is the nef cone $\operatorname{Nef}(X)$, which is dual to the Mori cone of curves (see for example [Lazarsfeld 2004]). In this paper, we will study the nef cone of the Hilbert scheme of points $X^{[n]}$, where $X$ is a smooth projective surface over $\mathbb{C}$.

Nef divisors on Hilbert schemes of points on surfaces $X^{[n]}$ are sometimes easy to construct by classical methods. If $L$ is an $(n-1)$-very ample line bundle

J. Huizenga was partially supported by an NSF Mathematical Sciences Postdoctoral Research Fellowship. B. Schmidt was partially supported by NSF grant DMS-1523496 (PI Emanuele Macrì) and a Presidential Fellowship of the Ohio State University.

MSC2010: primary 14C05; secondary 14E30, 14J29, 14J60.

Keywords: Hilbert schemes, surfaces, nef cone, ample cone, birational geometry, Bridgeland stability. 
on $X$, then for any $Z \in X^{[n]}$ we have an inclusion $H^{0}\left(L \otimes I_{Z}\right) \rightarrow H^{0}(L)$ which defines a morphism from $X^{[n]}$ to the Grassmannian $G\left(h^{0}(L)-n, h^{0}(L)\right)$. The pullback of an ample divisor on the Grassmannian is nef on $X^{[n]}$. It is frequently possible to construct extremal nef divisors by this method. For example, this method completely computes the nef cone of $X^{[n]}$ when $X$ is a del Pezzo surface of degree $\geq 2$ or a Hirzebruch surface (see [Arcara et al. 2013; Bertram and Coskun 2013]). Unfortunately, this approach to computing the nef cone is insufficient in general. At the very least, to study nef cones of more interesting surfaces it would be necessary to study an analog of $k$-very ampleness for higher rank vector bundles, which is considerably more challenging than line bundles.

More recently, many nef cones have been computed by making use of Bridgeland stability conditions and the positivity lemma of Bayer and Macrì (see [Bridgeland 2007; 2008; Arcara and Bertram 2013; Bayer and Macrì 2014b] for background on these topics, which will be reviewed in Section 2). Let $v=\operatorname{ch}\left(I_{Z}\right) \in K_{0}(X)$, where $Z \in X^{[n]}$. In the stability manifold $\operatorname{Stab}(X)$ for $X$ there is an open Gieseker chamber $\mathcal{C}$ such that if $\sigma \in \mathcal{C}$ then $M_{\sigma}(v) \cong X^{[n]}$, where $M_{\sigma}(v)$ is the moduli space of $\sigma$-semistable objects with invariants $\boldsymbol{v}$. The positivity lemma associates to any $\sigma \in \overline{\mathcal{C}}$ a nef divisor on $X^{[n]}$. Stability conditions in the boundary $\partial \mathcal{C}$ frequently give rise to extremal nef divisors. The positivity lemma also classifies the curves orthogonal to a nef divisor constructed in this way, and so gives a tool for checking extremality.

The stability manifold is rather large in general, so computation of the full Gieseker chamber can be unwieldy. We deal with this problem by focusing on a small slice of the stability manifold parametrized by a half-plane. Up to scale, the corresponding divisors in $N^{1}\left(X^{[n]}\right)$ form an affine ray. The nef cone $\operatorname{Nef}\left(X^{[n]}\right)$ is spanned by a codimension- 1 subcone identified with $\operatorname{Nef}(X)$ and other more interesting classes which are positive on curves contracted by the Hilbert-Chow morphism. Since $\operatorname{Nef}\left(X^{[n]}\right)$ is convex, we can study $\operatorname{Nef}\left(X^{[n]}\right)$ by looking at positivity properties of divisors along rays in $N^{1}\left(X^{[n]}\right)$ starting from a class in $\operatorname{Amp}(X) \subset \operatorname{Nef}\left(X^{[n]}\right)$. The positivity lemma gives us an effective criterion for testing when divisors along the ray are nef.

The slices of the stability manifold that we consider are given by a pair of divisors $(H, D)$ on $X$ with $H$ ample and $-D$ effective. The following is a weak version of one of our main theorems.

Theorem 1.1. Let $X$ be a smooth projective surface. If $n \gg 0$, then there is an extremal nef divisor on $X^{[n]}$ coming from the $(H, D)$-slice. It can be explicitly computed if both the intersection pairing on $\operatorname{Pic}(X)$ and the set of effective classes in $\operatorname{Pic}(X)$ are known. An orthogonal curve class is given by $n$ points moving in a $g_{n}^{1}$ on a curve of a particular class. 
See Section 3, especially Corollary 3.7 and Theorem 3.11, for more explicit statements. Stronger statements can also be shown under strong assumptions on $\operatorname{Pic}(X)$; for example, we study the Picard rank 1 case in detail in Section 4. Recall that if $X$ is a surface of irregularity $q:=H^{1}\left(\mathcal{O}_{X}\right)=0$, then $N^{1}\left(X^{[n]}\right)$ is spanned by the divisor $B$ of nonreduced schemes and divisors $L^{[n]}$ induced by divisors $L \in \operatorname{Pic}(X)$; see Section 2A for details.

Theorem 1.2. Let $X$ be a smooth projective surface with $\operatorname{Pic} X \cong \mathbb{Z} H$, where $H$ is an ample divisor. Let $a>0$ be the smallest integer such that $a H$ is effective. If

$$
n \geq \max \left\{a^{2} H^{2}, p_{a}(a H)+1\right\},
$$

then $\operatorname{Nef}\left(X^{[n]}\right)$ is spanned by the divisor $H^{[n]}$ and the divisor

$$
\frac{1}{2} K_{X}^{[n]}+\left(\frac{a}{2}+\frac{n}{a H^{2}}\right) H^{[n]}-\frac{1}{2} B .
$$

An orthogonal curve class is given by letting $n$ points move in a $g_{n}^{1}$ on a curve in $X$ of class $a H$.

Note that in the Picard rank 1 case the divisor class $(*)$ is frequently of the form $\lambda H^{[n]}-\frac{1}{2} B$ for a noninteger number $\lambda \in \mathbb{Q}$. Any divisor constructed from an $(n-1)$-very ample line bundle will be of the form $\lambda H^{[n]}-\frac{1}{2} B$ with $\lambda \in \mathbb{Z}$, so in general the edge of the nef cone cannot be obtained from line bundles in this way.

The required lower bound on $n$ in Theorem 1.2 can be improved in specific examples where special linear series on hyperplane sections are better understood.

Theorem 1.3. Let $X$ be one of the following surfaces:

(1) a very general hypersurface in $\mathbb{P}^{3}$ of degree $d \geq 4$, or

(2) a very general degree-d cyclic branched cover of $\mathbb{P}^{2}$ of general type.

In either case, $\operatorname{Pic}(X) \cong \mathbb{Z} H$ with $H$ effective. Suppose $n \geq d-1$ in the first case, and $n \geq d$ in the second case. Then $\operatorname{Nef}\left(X^{[n]}\right)$ is spanned by $H^{[n]}$ and the divisor class $(*)$ with $a=1$.

Finally, in Section 5 we compute the nef cone of $X^{[n]}$ where $X$ is a smooth del Pezzo surface of degree 1 and $n \geq 2$ is arbitrary. This computation was an open problem posed by Bertram and Coskun [2013]; they noted that the method of $k$-very ample line bundles would not be sufficient to prove the expected answer. Since $X$ has Picard rank 9, this computation makes full use of the general methods developed in Section 3. If $C \subset X$ is a reduced, irreducible curve which admits a $g_{n}^{1}$, we write $C_{[n]}$ for the curve in the Hilbert scheme $X^{[n]}$ given by letting $n$ points move in a $g_{n}^{1}$ on $C$.

Theorem 1.4. Let $X$ be a smooth del Pezzo surface of degree 1. The Mori cone of curves $\operatorname{NE}\left(X^{[n]}\right)$ is spanned by the 240 classes $E_{[n]}$ given by $(-1)$-curves $E \subset X$, 
the class of a curve contracted by the Hilbert-Chow morphism, and the class $F_{[n]}$, where $F \in\left|-K_{X}\right|$ is an anticanonical curve. The nef cone is determined by duality.

Many previous authors have used Bridgeland stability conditions to study nef cones and wall-crossing for Hilbert schemes $X^{[n]}$ and moduli spaces of sheaves $M_{H}(v)$ for various classes of surfaces. For instance, the program was studied for $\mathbb{P}^{2}$ in [Arcara et al. 2013; Coskun and Huizenga 2016; Bertram et al. 2014; Li and Zhao 2013], Hirzebruch and del Pezzo surfaces in [Bertram and Coskun 2013], abelian surfaces in [Yanagida and Yoshioka 2014; Maciocia and Meachan 2013], K3 surfaces in [Bayer and Macrì 2014b; 2014a; Hassett and Tschinkel 2010], and Enriques surfaces in [Nuer 2014]. Our results unify several of these approaches. Additionally, nef cones were classically studied in the context of $k$-very ample line bundles in papers such as [Ellingsrud et al. 2001; Beltrametti and Sommese 1991; Beltrametti et al. 1989; Catanese and Göttsche 1990].

\section{Preliminaries}

Throughout the paper, we let $X$ be a smooth projective surface over $\mathbb{C}$.

2A. Divisors and curves on $X^{[n]}$. For simplicity we assume that $X$ has irregularity $q=h^{1}\left(\mathcal{O}_{X}\right)=0$ in this subsection. By work of Fogarty [1968], the Hilbert scheme $X^{[n]}$ is a smooth projective variety of dimension $2 n$ which resolves the singularities in the symmetric product $X^{(n)}$ via the Hilbert-Chow morphism $X^{[n]} \rightarrow X^{(n)}$. A line bundle $L$ on $X$ induces the $S_{n}$-equivariant line bundle $L^{\otimes n}$ on $X^{n}$ which descends to a line bundle $L^{(n)}$ on the symmetric product $X^{(n)}$. The pullback of $L^{(n)}$ by the Hilbert-Chow morphism $X^{[n]} \rightarrow X^{(n)}$ defines a line bundle on $X^{[n]}$ which we will denote by $L^{[n]}$. Intuitively, if $L \cong \mathcal{O}_{X}(D)$ for a reduced effective divisor $D \subset X$, then $L^{[n]}$ can be represented by the divisor $D^{[n]}$ of schemes $Z \subset X$ which meet $D$.

Fogarty shows that

$$
\operatorname{Pic}\left(X^{[n]}\right) \cong \operatorname{Pic}(X) \oplus \mathbb{Z}(B / 2),
$$

where $\operatorname{Pic}(X) \subset \operatorname{Pic}\left(X^{[n]}\right)$ is embedded by $L \mapsto L^{[n]}$ and $B$ is the locus of nonreduced schemes, i.e., the exceptional divisor of the Hilbert-Chow morphism [Fogarty 1973]. Tensoring by the real numbers, the Néron-Severi space $N^{1}\left(X^{[n]}\right)$ is therefore spanned by $N^{1}(X)$ and $B$.

There are also curve classes in $X^{[n]}$ induced by curves in $X$. Two different constructions are immediate. Let $C \subset X$ be a reduced and irreducible curve.

(1) There is a curve $\tilde{C}_{[n]}$ in $X^{[n]}$ given by fixing $n-1$ general points of $X$ and letting an $n$-th point move along $C$.

(2) If $C$ admits a $g_{n}^{1}$, i.e., a degree- $n$ map to $\mathbb{P}^{1}$, then the fibers of $C \rightarrow \mathbb{P}^{1}$ give a rational curve $\mathbb{P}^{1} \rightarrow X^{[n]}$. We write $C_{[n]}$ for this class. 
These constructions preserve intersection numbers, in the sense that if $D \subset X$ is a divisor and $C \subset X$ is a curve then

$$
D^{[n]} \cdot \tilde{C}_{[n]}=D^{[n]} \cdot C_{[n]}=D \cdot C .
$$

Part of the nef cone $\operatorname{Nef}\left(X^{[n]}\right)$ is easily described in terms of the nef cone of $X$. If $D$ is an ample divisor, then $D^{(n)}$ is ample so $D^{[n]}$ is nef. In the limit, we find that if $D$ is nef then $D^{[n]}$ is nef. Conversely, if $D$ is not nef then there is an irreducible curve $C$ with $D \cdot C<0$, so $D^{[n]} \cdot \tilde{C}_{[n]}<0$ and $D^{[n]}$ is not nef. Under the Fogarty isomorphism,

$$
\operatorname{Nef}\left(X^{[n]}\right) \cap N^{1}(X)=\operatorname{Nef}(X) .
$$

The hyperplane $N^{1}(X) \subset N^{1}\left(X^{[n]}\right)$ is orthogonal to any curve contracted by the Hilbert-Chow morphism, so all the divisors in $\operatorname{Nef}(X) \subset \operatorname{Nef}\left(X^{[n]}\right)$ are extremal. Since $B$ is the exceptional locus of the Hilbert-Chow morphism, we see that any nef class must have nonpositive coefficient of $B$. After scaling, then, we see that computation of the cone $\operatorname{Nef}\left(X^{[n]}\right)$ reduces to describing the nef classes of the form $L^{[n]}-\frac{1}{2} B$ lying outside $\operatorname{Nef}(X) \subset \operatorname{Nef}\left(X^{[n]}\right)$.

2B. Bridgeland stability conditions. We now recall some basic definitions and properties of Bridgeland stability conditions. We fix a polarization $H \in \operatorname{Pic}(X)_{\mathbb{R}}$. For any divisor $D \in \operatorname{Pic}(X)_{\mathbb{R}}$, the twisted Chern character $\mathrm{ch}^{D}=e^{-D} \mathrm{ch}$ can be expanded as

$$
\operatorname{ch}_{0}^{D}=\mathrm{ch}_{0}, \quad \operatorname{ch}_{1}^{D}=\mathrm{ch}_{1}-D \mathrm{ch}_{0}, \quad \operatorname{ch}_{2}^{D}=\mathrm{ch}_{2}-D \cdot \mathrm{ch}_{1}+\frac{1}{2} D^{2} \mathrm{ch}_{0} .
$$

Recall that a Bridgeland stability condition is a pair $\sigma=(Z, \mathcal{A})$ for which $Z: K_{0}(X) \rightarrow \mathbb{C}$ is an additive homomorphism and $\mathcal{A} \subset D^{b}(X)$ is the heart of a bounded t-structure. In particular, $\mathcal{A}$ is an abelian category. Moreover, $Z$ maps any nontrivial object in $\mathcal{A}$ to the upper half-plane or the negative real line. The $\sigma$-slope function is defined by

$$
v_{\sigma}=-\frac{\mathfrak{R} Z}{\Im Z},
$$

and $\sigma$-(semi)stability of objects of $\mathcal{A}$ is defined in terms of this slope function. More technical requirements are the existence of Harder-Narasimhan filtrations and the support property. We recommend [Bridgeland 2007] for a more precise definition. The support property is well explained in Appendix A of [Bayer et al. 2014].

In the case of surfaces, Bridgeland [2008] and Arcara and Bertram [2013] showed how to construct Bridgeland stability conditions in a slice corresponding to a choice of an ample divisor $H \in \operatorname{Pic}(X)_{\mathbb{R}}$ and arbitrary twisting divisor $D \in \operatorname{Pic}(X)_{\mathbb{R}}$. The 
912 B. Bolognese, J. Huizenga, Y. Lin, E. Riedl, B. Schmidt, M. Woolf and X. Zhao

classical Mumford slope function for twisted Chern characters is defined by

$$
\mu_{H, D}=\frac{H \cdot \operatorname{ch}_{1}^{D}}{H^{2} \operatorname{ch}_{0}^{D}},
$$

where torsion sheaves are interpreted as having positive infinite slope. Given a real number $\beta \in \mathbb{R}$, there are two categories defined as

$$
\begin{aligned}
& \mathcal{T}_{\beta}=\left\{E \in \operatorname{Coh}(X): \text { any quotient } E \rightarrow G \text { satisfies } \mu_{H, D}(G)>\beta\right\}, \\
& \mathcal{F}_{\beta}=\left\{E \in \operatorname{Coh}(X): \text { any subsheaf } F \hookrightarrow E \text { satisfies } \mu_{H, D}(F) \leq \beta\right\} .
\end{aligned}
$$

A new heart of a bounded t-structure is defined as the extension closure $\mathcal{A}_{\beta}:=$ $\left\langle\mathcal{F}_{\beta}[1], \mathcal{T}_{\beta}\right\rangle$. We fix an additional positive real number $\alpha$ and define the homomorphism as

$$
Z_{\beta, \alpha}=-\operatorname{ch}_{2}^{D+\beta H}+\frac{1}{2} \alpha^{2} H^{2} \operatorname{ch}_{0}^{D+\beta H}+i H \cdot \mathrm{ch}_{1}^{D+\beta H} .
$$

The pair $\sigma_{\beta, \alpha}:=\left(Z_{\beta, \alpha}, \mathcal{A}_{\beta}\right)$ is then a Bridgeland stability condition. The $(H, D)$ slice of stability conditions is the family of stability conditions

$$
\left\{\sigma_{\beta, \alpha}: \beta, \alpha \in \mathbb{R}, \alpha>0\right\}
$$

parametrized by the $(\beta, \alpha)$ upper half-plane.

Definition 2.1. Fix a set of invariants $v \in K_{0}(X)$.

(1) Let $\boldsymbol{w} \in K_{0}(X)$ be a vector such that $\boldsymbol{v}$ and $\boldsymbol{w}$ do not have the same $\sigma_{\beta, \alpha}$-slope everywhere in the $(H, D)$-slice. The numerical wall for $\boldsymbol{v}$ given by $\boldsymbol{w}$ is the set of points $(\beta, \alpha)$ where $\boldsymbol{v}$ and $\boldsymbol{w}$ have the same $\sigma_{\beta, \alpha}$-slope.

(2) A numerical wall for $\boldsymbol{v}$ given by a vector $\boldsymbol{w}$ as above is a wall (or actual wall) if there is a point $(\beta, \alpha)$ on the wall and an exact sequence $0 \rightarrow F \rightarrow E \rightarrow G \rightarrow 0$ in $\mathcal{A}_{\beta}$, where $\operatorname{ch} F=\boldsymbol{w}$, ch $E=\boldsymbol{v}$, and $F, E$, and $G$ are $\sigma_{\beta, \alpha}$-semistable objects (of the same $\sigma_{\beta, \alpha}$-slope).

We write $K_{\text {num }}(X)$ for the numerical Grothendieck group of classes in $K_{0}(X)$ modulo numerical equivalence. Note that numerical walls for $v \in K_{0}(X)$ only depend on the numerical class of $\boldsymbol{v}$, while actual walls a priori depend on $c_{1}(\boldsymbol{v}) \in$ $\operatorname{Pic}(X)$. The structure of walls in a slice is heavily restricted by Bertram's nested wall theorem. This was first observed for Picard rank 1 with $D=0$, but the proof immediately generalizes by replacing $\mathrm{ch}$ by $\mathrm{ch}^{D}$ everywhere.

Theorem 2.2 [Maciocia 2014]. Let $\boldsymbol{v} \in K_{0}(X)$.

(1) Numerical walls for $\boldsymbol{v}$ can either be semicircles with center on the $\beta$-axis or the unique vertical line given by $\beta=\mu_{H, D}(v)$. Moreover, the apex of each semicircle lies on the hyperbola $\Re Z_{\beta, \alpha}(v)=0$. 
(2) Numerical walls for $\boldsymbol{v}$ are disjoint, and the semicircular walls on either side of the vertical wall are nested.

(3) If $W_{1}$ and $W_{2}$ are two semicircular numerical walls left of the vertical wall with centers $\left(s_{W_{1}}, 0\right)$ and $\left(s_{W_{2}}, 0\right)$, then $W_{2}$ is nested inside $W_{1}$ if and only if $s_{W_{1}}<s_{W_{2}}$.

(4) Suppose $0 \rightarrow F \rightarrow E \rightarrow G \rightarrow 0$ is an exact sequence destabilizing an object $E$ with $\operatorname{ch}(E)=v$ at a point $(\beta, \alpha)$ on a numerical wall $W$, in the sense that all three objects have the same $\sigma_{\beta, \alpha}$-slope and this is an exact sequence in $\mathcal{A}_{\beta}$. Then it is an exact sequence of objects in $\mathcal{A}_{\beta^{\prime}}$ with the same $\sigma_{\beta^{\prime}, \alpha^{\prime}}$-slope for all $\left(\beta^{\prime}, \alpha^{\prime}\right) \in W$. That is, $E$ is destabilized along the entire wall.

2C. Slope and discriminant. The explicit geometry of walls is frequently best understood in terms of slopes and discriminants; the formulas presented here previously appeared in [Coskun and Huizenga 2014] in the context of $\mathbb{P}^{2}$. When the rank is nonzero, we define

$$
\Delta_{H, D}=\frac{1}{2} \mu_{H, D}^{2}-\frac{\operatorname{ch}_{2}^{D}}{H^{2} \operatorname{ch}_{0}^{D}} .
$$

The Bogomolov inequality gives $\Delta_{H, D}(E) \geq 0$ whenever $E$ is an $(H, D)$-twisted Gieseker semistable sheaf. Observe that $\Delta_{H, D+\beta H}=\Delta_{H, D}$ for every $\beta \in \mathbb{R}$. A straightforward calculation shows that for vectors of nonzero rank the slope function for the stability condition $\sigma_{\beta, \alpha}$ in the $(H, D)$-slice is given by

$$
v_{\sigma_{\beta, \alpha}}=\frac{\left(\mu_{H, D}-\beta\right)^{2}-\alpha^{2}-2 \Delta_{H, D}}{\left(\mu_{H, D}-\beta\right)} .
$$

Suppose $\boldsymbol{v}, \boldsymbol{w}$ are two classes with positive rank, and let their slopes and discriminants be $\mu_{H, D}, \Delta_{H, D}$ and $\mu_{H, D}^{\prime}, \Delta_{H, D}^{\prime}$, respectively. The numerical wall $W$ in the $(H, D)$-slice where $\boldsymbol{v}$ and $\boldsymbol{w}$ have the same slope is computed as follows.

- If $\mu_{H, D}=\mu_{H, D}^{\prime}$ and $\Delta_{H, D}=\Delta_{H, D}^{\prime}$, then $\boldsymbol{v}$ and $\boldsymbol{w}$ have the same slope everywhere in the slice, so there is no numerical wall.

- If $\mu_{H, D}=\mu_{H, D}^{\prime}$ and $\Delta_{H, D} \neq \Delta_{H, D}^{\prime}$, then $W$ is the vertical wall $\beta=\mu_{H, D}$.

- If $\mu_{H, D} \neq \mu_{H, D}^{\prime}$, then (1) implies $W$ is the semicircle with center $\left(s_{W}, 0\right)$ and radius $\rho_{W}$, where

$$
\begin{aligned}
& s_{W}=\frac{1}{2}\left(\mu_{H, D}+\mu_{H, D}^{\prime}\right)-\frac{\Delta_{H, D}-\Delta_{H, D}^{\prime}}{\mu_{H, D}-\mu_{H, D}^{\prime}}, \\
& \rho_{W}^{2}=\left(s_{W}-\mu_{H, D}\right)^{2}-2 \Delta_{H, D},
\end{aligned}
$$

provided that the expression defining $\rho_{W}^{2}$ is positive; if it is negative then the wall is empty. 
914 B. Bolognese, J. Huizenga, Y. Lin, E. Riedl, B. Schmidt, M. Woolf and X. Zhao

Notice that if $\Delta_{H, D}(v) \geq 0$ then numerical walls for $\boldsymbol{v}$ left of the vertical wall accumulate at the point

$$
\left(\mu_{H, D}(\boldsymbol{v})-\sqrt{2 \Delta_{H, D}(\boldsymbol{v})}, 0\right)
$$

as their radii go to 0 .

2D. Nef divisors and the positivity lemma. In this section, we describe the positivity lemma of Bayer and Macrì. Let $\sigma=(Z, \mathcal{A})$ be a stability condition on $X$, $\boldsymbol{v} \in K_{\text {num }}(X)$ and $S$ a proper algebraic space of finite type over $\mathbb{C}$. Let $\mathcal{E} \in D^{b}(X \times S)$ be a flat family of $\sigma$-semistable objects of class $\boldsymbol{v}$, i.e., for every $\mathbb{C}$-point $p \in S$, the derived restriction $\left.\mathcal{E}\right|_{\pi_{S}^{-1}(\{p\})}$ is $\sigma$-semistable of class $\boldsymbol{v}$. Then Bayer and Macrì define a numerical divisor class $D_{\sigma, \mathcal{E}} \in N^{1}(S)$ on the space $S$ by assigning its intersection with any projective integral curve $C \subset S$ :

$$
D_{\sigma, \mathcal{E}} \cdot C=\Im\left(-\frac{Z\left(\left.\left(p_{X}\right)_{*} \mathcal{E}\right|_{C \times X}\right)}{Z(\boldsymbol{v})}\right) .
$$

The positivity lemma shows that this divisor inherits positivity properties from the homomorphism $Z$, and classifies the curve classes orthogonal to the divisor. Recall that two $\sigma$-semistable objects are $S$-equivalent with respect to $\sigma$ if their sets of Jordan-Hölder factors are the same.

Theorem 2.3 (positivity lemma [Bayer and Macrì 2014b, Lemma 3.3]). The divisor $D_{\sigma, \mathcal{E}} \in N^{1}(S)$ is nef. Moreover, if $C \subset S$ is a projective integral curve then $D_{\sigma, \mathcal{E}} \cdot C=0$ if and only if two general objects parametrized by $C$ are $S$-equivalent with respect to $\sigma$.

Our primary use of the positivity lemma is to attempt to construct extremal nef divisors on Hilbert schemes of points. Thus it is important to recover Hilbert schemes of points as Bridgeland moduli spaces. Recall that a torsion-free coherent sheaf $E$ is $(H, D)$-twisted Gieseker semistable if for every $F \subset E$ we have

$$
\frac{\chi\left(F \otimes \mathcal{O}_{X}(m H-D)\right)}{\operatorname{rk}(F)} \leq \frac{\chi\left(E \otimes \mathcal{O}_{X}(m H-D)\right)}{\operatorname{rk}(E)}
$$

for all $m \gg 0$, where the Euler characteristic is computed formally via RiemannRoch; see [Matsuki and Wentworth 1997]. For any class $v \in K_{0}(X)$, there are projective moduli spaces $M_{H, D}(v)$ of $S$-equivalence classes of $(H, D)$-twisted Gieseker semistable sheaves with class $\boldsymbol{v}$. If $\boldsymbol{v}=(1,0,-n)$ is the Chern character of an ideal sheaf of $n$ points then $M_{H, D}(v)=X^{[n]}$. Note that if the irregularity of $X$ is nonzero, then it is crucial to fix the determinant.

Fix an $(H, D)$-slice in the stability manifold, and fix a vector $v \in K_{0}(X)$ with positive rank. If $\beta$ lies to the left of the vertical wall $\beta=\mu_{H, D}(\boldsymbol{v})$ for $\boldsymbol{v}$, then for $\alpha \gg 0$ the moduli space coincides with a twisted Gieseker moduli space. 
Proposition 2.4 (the large volume limit [Bridgeland 2008; Maciocia 2014]). Fix divisors $(H, D)$ giving a slice in $\operatorname{Stab}(X)$. Let $v \in K_{0}(X)$ be a vector with positive rank, and let $\beta \in \mathbb{R}$ be such that $\mu_{H, D}(\boldsymbol{v})>\beta$. If $E \in \mathcal{A}_{\beta}$ has $\operatorname{ch}(E)=\boldsymbol{v}$ then $E$ is $\sigma_{\beta, \alpha}$-semistable for all $\alpha \gg 0$ if and only if $E$ is an $\left(H, D-\frac{1}{2} K_{X}\right)$-twisted Gieseker semistable sheaf.

Moreover, in the quadrant of the $(H, D)$-slice left of the vertical wall there is a largest semicircular wall for $\boldsymbol{v}$, called the Gieseker wall. For all $(\beta, \alpha)$ between this wall and the vertical wall, the moduli space $M_{\sigma_{\beta, \alpha}}(\boldsymbol{v})$ coincides with the moduli space $M_{H, D-K_{X} / 2}(v)$ of $\left(H, D-\frac{1}{2} K_{X}\right)$-twisted Gieseker semistable sheaves.

We use these results as follows. Let $v=(1,0,-n) \in K_{0}(X)$ be the vector for the Hilbert scheme $X^{[n]}$, and let $\sigma_{+}$be a stability condition in the $(H, D)$-slice lying above the Gieseker wall, so that $M_{\sigma_{+}}(v) \cong X^{[n]}$. Let $\mathcal{E} /\left(X \times X^{[n]}\right)$ be the universal ideal sheaf, and let $\sigma_{0}$ be a stability condition on the Gieseker wall. By the definition of the Gieseker wall, $\mathcal{E}$ is a family of $\sigma_{0}$-semistable objects, so there is an induced nef divisor $D_{\sigma_{0}, \mathcal{E}}$ on $X^{[n]}$. Furthermore, curves orthogonal to $D_{\sigma_{0}, \mathcal{E}}$ are understood in terms of destabilizing sequences along the wall, so it is possible to test for extremality.

\section{Gieseker walls and the nef cone}

Fix an ample divisor $H \in \operatorname{Pic}(X)$ with $H^{2}=d$ and an antieffective divisor $D$. In this section we study the nef divisor arising from the Gieseker wall (i.e., the largest wall where some ideal sheaf is destabilized) in the slice of the stability manifold given by the pair $(H, D)$. We first compute the Gieseker wall, and then investigate when the corresponding nef divisor is in fact extremal.

3A. Bounding higher rank walls. The main difficulty in computing extremal rays of the nef cone is to show that a destabilizing subobject along the Gieseker wall is a line bundle, and not some higher rank sheaf. We first prove a lemma which generalizes Proposition 8.3 of [Coskun and Huizenga 2016] from $X=\mathbb{P}^{2}$ to an arbitrary surface. We prove the result in slightly more generality than we will need here as we expect it to be useful in future work.

Lemma 3.1. Let $\sigma_{0}$ be a stability condition in the $(H, D)$-slice, and suppose

$$
0 \rightarrow F \rightarrow E \rightarrow G \rightarrow 0
$$

is an exact sequence of $\sigma_{0}$-semistable objects of the same $\sigma_{0}$-slope, where $E$ is an $(H, D)$-twisted Gieseker semistable torsion-free sheaf. If the map $F \rightarrow E$ of sheaves is not injective, then the radius $\rho_{W}$ of the wall $W$ defined by this sequence satisfies

$$
\rho_{W}^{2} \leq \frac{(\min \{\operatorname{rk}(F)-1, \operatorname{rk}(E)\})^{2}}{2 \operatorname{rk}(F)} \Delta_{H, D}(E) .
$$


Proof. The proof is similar to the proof in [Coskun and Huizenga 2016] given in the case of $\mathbb{P}^{2}$; we present it for completeness. The object $F$ is a torsion-free sheaf by the standard cohomology sequence and the fact that the heart of the t-structure in the slice we are working in consists of objects which only have nonzero cohomology sheaves in degrees 0 and -1 . The exact sequence along $W$ gives an exact sequence

$$
0 \rightarrow K \rightarrow F \rightarrow E \rightarrow C \rightarrow 0
$$

of sheaves of ranks $k, f, e, c$, respectively. By assumption, $k, f, e>0$. Let $\left(s_{W}, 0\right)$ be the center of $W$. As $F$ is in the category $\mathcal{T}_{\beta}$ whenever $(\beta, \alpha)$ is on $W$, we find that $\mu_{H, D}(F) \geq s_{W}+\rho_{W}$, so

$$
\begin{aligned}
d f\left(s_{W}+\rho_{W}\right) & \leq d f \mu_{H, D}(F)=\operatorname{ch}_{1}^{D}(F) \cdot H=\left(\operatorname{ch}_{1}^{D}(K)+\operatorname{ch}_{1}^{D}(E)-\operatorname{ch}_{1}^{D}(C)\right) \cdot H \\
& =d k \mu_{H, D}(K)+d e \mu_{H, D}(E)-\operatorname{ch}_{1}^{D}(C) \cdot H .
\end{aligned}
$$

Similarly, $K \in \mathcal{F}_{\beta}$ along $W$, so $\mu_{H, D}(K) \leq s_{W}-\rho_{W}$ and

$$
d f\left(s_{W}+\rho_{W}\right) \leq d k\left(s_{W}-\rho_{W}\right)+d e \mu_{H, D}(E)-\operatorname{ch}_{1}^{D}(C) \cdot H,
$$

which gives

$$
d(k+f) \rho_{W} \leq d(k-f) s_{W}+d e \mu_{H, D}(E)-\operatorname{ch}_{1}^{D}(C) \cdot H .
$$

We now wish to eliminate the term $\operatorname{ch}_{1}^{D}(C) \cdot H$ in inequality (5). If $C$ is either 0 or torsion, then $\operatorname{ch}_{1}^{D}(C) \cdot H \geq 0$ and $-e=k-f$, and we deduce

$$
(k+f) \rho_{W} \leq(k-f)\left(s_{W}-\mu_{H, D}(E)\right) .
$$

Suppose instead that $C$ is not torsion. Since $C$ is a quotient of the semistable sheaf $E$, we have $\mu_{H, D}(C) \geq \mu_{H, D}(E)$, $\operatorname{so~} \operatorname{ch}_{1}^{D}(C) \cdot H=d c \mu_{H, D}(C) \geq d c \mu_{H, D}(E)$. As $k-f=c-e$, we find that inequality (6) also holds in this case.

Both sides of inequality (6) are positive, so squaring both sides gives

$$
(k+f)^{2} \rho_{W}^{2} \leq(k-f)^{2}\left(s_{W}-\mu_{H, D}(E)\right)^{2} .
$$

Formula (3) for $\rho_{W}^{2}$ shows this is equivalent to

$$
(k+f)^{2} \rho_{W}^{2} \leq(k-f)^{2}\left(\rho_{W}^{2}+2 \Delta_{H, D}(E)\right),
$$

from which we obtain

$$
\rho_{W}^{2} \leq \frac{(k-f)^{2}}{2 k f} \Delta_{H, D}(E) .
$$

Since $k=f-e+c$, we see that $k \geq \max \{1, f-e\}$. By taking derivatives in $k$, we see that $(k-f)^{2} /(2 k f)$ is decreasing for $k+f>0$, and so the maximum possible value of the right-hand side must occur when $k=\max \{1, f-e\}$. The denominator 
will be at least $2 f$ in this case, and the numerator is $\min \left\{(f-1)^{2}, e^{2}\right\}$. The result follows.

For our present work we will only need the next consequence of Lemma 3.1, which follows immediately from computing $\Delta_{H, D}\left(I_{Z}\right)$.

Corollary 3.2. With the hypotheses of Lemma 3.1, if $E$ is an ideal sheaf $I_{Z} \in X^{[n]}$ and $F$ has rank at least 2 , then the radius of the corresponding wall satisfies

$$
\rho_{W}^{2} \leq \frac{2 n d+(H \cdot D)^{2}-d D^{2}}{8 d^{2}}:=\varrho_{H, D, n} .
$$

The number $\varrho_{H, D, n}$ therefore bounds the squares of the radii of higher rank walls for $X^{[n]}$.

3B. Rank-1 walls and critical divisors. In the cases where we compute the Gieseker wall, the ideal sheaf that is destabilized along the wall will be destabilized by a rank-1 subobject. We first compute the numerical walls given by rank-1 subobjects.

Lemma 3.3. Consider a rank-1 torsion-free sheaf $F=I_{Z^{\prime}}(-L)$, where $Z^{\prime}$ is a zero-dimensional scheme of length $w$ and $L$ is an effective divisor. In the $(H, D)$ slice, the numerical wall $W$ for $X^{[n]}$ where $F$ has the same slope as an ideal $I_{Z}$ of $n$ points has center $\left(s_{W}, 0\right)$ given by

$$
s_{W}=-\frac{2(n-w)+L^{2}+2(D \cdot L)}{2(H \cdot L)} .
$$

Proof. This is an immediate consequence of (2) for the center of a wall.

Recalling that walls for $X^{[n]}$ left of the vertical wall get larger as their centers decrease, we deduce the following consequence.

Lemma 3.4. If the Gieseker wall in the $(H, D)$-slice is given by a rank-1 subobject, then it is a line bundle $\mathcal{O}_{X}(-L)$ for some effective divisor $L$.

Proof. Suppose some $I_{Z} \in X^{[n]}$ is destabilized along the Gieseker wall $W$ by a sheaf of the form $I_{Z^{\prime}}(-L)$, where $Z^{\prime}$ is a nonempty zero-dimensional scheme and $L$ is effective. By Lemma 3.3, the numerical wall $W^{\prime}$ given by $\mathcal{O}_{X}(-L)$ is strictly larger than $W$. Since $\mathcal{O}_{X}(-L)$ has the same $\mu_{H, D^{-}}$-slope as $I_{Z^{\prime}}(-L)$, which is in the categories along $W$, we find that $\mathcal{O}_{X}(-L)$ is in at least some of the categories along $W^{\prime}$. But then $W^{\prime}$ is an actual wall, since any ideal sheaf $I_{Z}$ where $Z$ lies on a curve $C \in|L|$ is destabilized along it. This contradicts the fact that $W$ is the Gieseker wall.

Less trivially, there is a further minimality condition automatically satisfied by a line bundle $\mathcal{O}_{X}(-L)$ which gives the Gieseker wall. We define the set of critical effective divisors with respect to $H$ and $D$ by

$\operatorname{CrDiv}(H, D)=\{-D\} \cup\{L \in \operatorname{Pic}(X)$ effective $: H \cdot L<H \cdot(-D)\}$. 
By [Hartshorne 1977, Exercise V.1.11], the set $\operatorname{CrDiv}(H, D) / \sim$ of critical divisors modulo numerical equivalence is finite. Therefore, the set of numerical walls for $X^{[n]}$ given by line bundles $\mathcal{O}_{X}(-L)$ with $L \in \operatorname{CrDiv}(H, D)$ is also finite. Note that the inequality $H \cdot L<H \cdot(-D)$ is equivalent to the inequality $\mu_{H, D}\left(\mathcal{O}_{X}(-L)\right)>0$. The next proposition demonstrates the importance of critical divisors.

Proposition 3.5. Assume $2 n>D^{2}$, and suppose the subobject giving the Gieseker wall for $X^{[n]}$ in the $(H, D)$-slice is a line bundle. Then the Gieseker wall is computed by $\mathcal{O}_{X}(-L)$, where $L \in \operatorname{CrDiv}(H, D)$ is chosen so that the numerical wall given by $\mathcal{O}_{X}(-L)$ is as large as possible.

Proof. First, consider the numerical wall $W$ given by $\mathcal{O}_{X}(D)$. By Lemma 3.3, the center $\left(s_{W}, 0\right)$ has

$$
s_{W}=\frac{2 n-D^{2}}{2(H \cdot D)}<0,
$$

since $2 n>D^{2}$ and $D$ is antieffective. Since $\mu_{H, D}\left(\mathcal{O}_{X}(D)\right)=\Delta_{H, D}\left(\mathcal{O}_{X}(D)\right)=0$, formula (3) for the radius of $W$ gives $\rho_{W}^{2}=s_{W}^{2}$. In particular, $W$ is nonempty, and $\mathcal{O}_{X}(D)$ lies in at least some of the categories along $W$. Since $D$ is antieffective, there are exact sequences of the form

$$
0 \rightarrow \mathcal{O}_{X}(D) \rightarrow I_{Z} \rightarrow I_{Z \subset C} \rightarrow 0,
$$

where $C \in|-D|$ and $Z \subset C$ is a collection of $n$ points. If no actual wall is larger than $W$, it follows that $W$ is an actual wall and it is the Gieseker wall.

Suppose the Gieseker wall is larger than $W$ and computed by a line bundle $\mathcal{O}_{X}(-L)$ with $L$ effective. Since $W$ passes through the origin in the $(\beta, \alpha)$ plane, $\mathcal{O}_{X}(-L)$ must lie in the category $\mathcal{T}_{0}$. Therefore $\mu_{H, D}\left(\mathcal{O}_{X}(-L)\right)>0$, and $L \in \operatorname{CrDiv}(H, D)$.

Conversely, suppose $L \in \operatorname{CrDiv}(H, D)$ is chosen to maximize the wall $W^{\prime}$ given by $\mathcal{O}_{X}(-L)$. Then no actual wall is larger than $W^{\prime}$. Since $s_{W}<0$ and $\mu_{H, D}\left(\mathcal{O}_{X}(-L)\right) \geq 0$, we find that $\mathcal{O}_{X}(-L)$ is in at least some of the categories along $W$, and hence in at least some of the categories along $W^{\prime}$. We conclude that $W^{\prime}$ is an actual wall, and therefore that it is the Gieseker wall.

Combining Corollary 3.2 and Proposition 3.5 gives our primary tool to compute the Gieseker wall.

Theorem 3.6. Assume $2 n>D^{2}$, and let $L \in \operatorname{CrDiv}(H, D)$ be a critical divisor such that the wall for $X^{[n]}$ given by $\mathcal{O}_{X}(-L)$ is as large as possible. If this wall has radius $\rho$ satisfying $\rho^{2} \geq \varrho_{H, D, n}$, then it is the Gieseker wall.

Conversely, if the Gieseker wall has radius satisfying $\rho^{2} \geq \varrho_{H, D, n}$ then it is obtained in this way. 
While the theorem is our sharpest result, it is useful to lose some generality to get a more explicit version. Since $-D \in \operatorname{CrDiv}(H, D)$, if the wall given by $\mathcal{O}_{X}(D)$ satisfies $\rho^{2} \geq \varrho_{H, D, n}$ then the Gieseker wall is computed by Theorem 3.6. This allows us to compute the Gieseker wall so long as $n$ is large enough, depending only on the intersection numbers of $H$ and $D$.

Corollary 3.7. Let

$$
\eta_{H, D}:=\frac{(H \cdot D)^{2}+d D^{2}}{2 d} .
$$

If $n \geq \eta_{H, D}$ then the Gieseker wall is the largest wall given by a critical divisor.

Furthermore, if $n>\eta_{H, D}$ then every $I_{Z}$ destabilized along the Gieseker wall fits into an exact sequence

$$
0 \rightarrow \mathcal{O}_{X}(-C) \rightarrow I_{Z} \rightarrow I_{Z \subset C} \rightarrow 0
$$

for some curve $C \in|L|$, where $L$ is a critical divisor computing the Gieseker wall. If the critical divisor computing the Gieseker wall is unique, then $\mathcal{O}_{X}(-C)$ and $I_{Z \subset C}$ are the Jordan-Hölder factors of any $I_{Z}$ destabilized along the Gieseker wall.

Proof. Observe that the inequality $n \geq \eta_{H, D}$ automatically implies the inequality $2 n>D^{2}$ needed to apply Theorem 3.6.

Let $W$ be the wall for $X^{[n]}$ in the $(H, D)$-slice corresponding to $\mathcal{O}_{X}(D)$. The center $\left(s_{W}, 0\right)$ of $W$ was computed in (7), and $\rho_{W}^{2}=s_{W}^{2}$. We find that $\rho_{W}^{2} \geq \varrho_{H, D, n}$ holds when $n \geq \eta_{H, D}$, with strict inequality when $n>\eta_{H, D}$.

When $n>\eta_{H, D}$ there can be no higher-rank destabilizing subobject of an $I_{Z}$ destabilized along the Gieseker wall, so there is an exact sequence as claimed. Furthermore, if there is only one critical divisor computing the wall, then there is a unique destabilizing subobject along the wall, so the Jordan-Hölder filtration has length two.

3C. Classes of divisors. In this subsection we give an elementary computation of the class of the divisor corresponding to a wall in a given slice of the stability manifold. Similar results have been obtained by Liu [2015], but the result is critical to our discussion so we include the proof. See [Bayer and Macrì 2014b, §4] for more details on the definitions and results we use here.

Throughout this subsection, let $v \in K_{0}(X)$ be a vector such that the moduli space $M_{H, D}(v)$ of $(H, D)$-Gieseker semistable sheaves admits a (quasi-)universal family $\mathcal{E}$ which is unique up to equivalence (Hilbert schemes $X^{[n]}$ are examples of such spaces). We also let $\sigma=(Z, \mathcal{A})$ be a stability condition in the closure of the Gieseker chamber for $\boldsymbol{v}$ in the $(H, D)$-slice. Then there is a well-defined corresponding divisor $D_{\sigma} \in N^{1}\left(M_{H, D-K_{X} / 2}(v)\right)$ which is independent of the choice of $\mathcal{E}$. 
Let $(\boldsymbol{v}, \boldsymbol{w})=\chi(\boldsymbol{v} \cdot \boldsymbol{w})$ be the Euler pairing on $K_{\text {num }}(X)_{\mathbb{R}}$, and write $\boldsymbol{v}^{\perp} \subset K_{\text {num }}(X)_{\mathbb{R}}$ for the orthogonal complement with respect to this pairing. The correspondence between stability conditions and divisor classes is understood in terms of the Donaldson homomorphism

$$
\lambda: \boldsymbol{v}^{\perp} \rightarrow N^{1}\left(M_{H, D-K_{X} / 2}(\boldsymbol{v})\right) .
$$

Since the Euler pairing is nondegenerate, there is a unique vector $\boldsymbol{w}_{\sigma} \in \boldsymbol{v}^{\perp}$ such that

$$
\mathfrak{I}\left(-\frac{Z\left(\boldsymbol{w}^{\prime}\right)}{Z(\boldsymbol{v})}\right)=\left(\boldsymbol{w}^{\prime}, \boldsymbol{w}_{\sigma}\right),
$$

for all $\boldsymbol{w}^{\prime} \in K_{\text {num }}(X)_{\mathbb{R}}$. Bayer and Macrì show that $D_{\sigma}=\lambda\left(\boldsymbol{w}_{\sigma}\right)$. In what follows, we write vectors in $K_{\text {num }}(X)_{\mathbb{R}}$ as $\left(\mathrm{ch}_{0}, \mathrm{ch}_{1}, \mathrm{ch}_{2}\right)$.

Proposition 3.8. With the above assumptions, suppose $\sigma$ lies on a numerical wall $W$ in the $(H, D)$-slice with center $\left(s_{W}, 0\right)$. Then $\boldsymbol{w}_{\sigma}$ is a multiple of

$$
\left(-1,-\frac{1}{2} K_{X}+s_{W} H+D, m\right) \in v^{\perp},
$$

where $m$ is determined by the requirement $\boldsymbol{w}_{\sigma} \in \boldsymbol{v}^{\perp}$.

In particular, if $X$ has irregularity 0 and $v=(1,0,-n)$ is the vector for $X^{[n]}$, then the divisor $D_{\sigma}$ is a multiple of

$$
\frac{1}{2} K_{X}^{[n]}-s_{W} H^{[n]}-D^{[n]}-\frac{1}{2} B .
$$

Remark 3.9. Suppose $X$ has irregularity 0 . Up to scale, the divisors induced by stability conditions in the $(H, D)$-slice give a ray in $N^{1}\left(X^{[n]}\right)$ emanating from the class $H^{[n]} \in \operatorname{Nef}(X) \subset \operatorname{Nef}\left(X^{[n]}\right)$. The particular ray is determined by the choice of the twisting divisor $D$.

Proof of Proposition 3.8. Since $\sigma$ is in the $(H, D)$-slice, write $\sigma=\sigma_{\beta, \alpha}$ and $(Z, \mathcal{A})=\left(Z_{\beta, \alpha}, \mathcal{A}_{\beta}\right)$ for short. Put $z=-1 / Z(\boldsymbol{v})=u+i v$. We evaluate the identity

$$
\Im\left(z Z\left(\boldsymbol{w}^{\prime}\right)\right)=\left(\boldsymbol{w}^{\prime}, \boldsymbol{w}_{\sigma}\right)
$$

defining $\boldsymbol{w}_{\sigma}$ on various classes $\boldsymbol{w}^{\prime}$ to compute $\boldsymbol{w}_{\sigma}$.

Write the Chern character $\boldsymbol{w}_{\sigma}=(r, C, d)$. Then

$$
-v=\mathfrak{s}(z Z(0,0,1))=\left((0,0,1), \boldsymbol{w}_{\sigma}\right)=r,
$$

so $r=-v$. Next, for any curve class $C^{\prime}$,

$$
\begin{aligned}
(u+\beta v)\left(C^{\prime} \cdot H\right)+v\left(C^{\prime} \cdot D\right) & =\Im\left(z Z\left(0, C^{\prime}, 0\right)\right) \\
& =\left(\left(0, C^{\prime}, 0\right), \boldsymbol{w}_{\sigma}\right)=\chi\left(0,-v C^{\prime}, C^{\prime} \cdot C\right) .
\end{aligned}
$$


By Riemann-Roch and adjunction,

$$
\begin{aligned}
\chi\left(0,-v C^{\prime}, C^{\prime} \cdot C\right)=-v\left(\left(-\frac{1}{v}\left(C^{\prime} \cdot C\right)+\frac{1}{2}\left(C^{\prime}\right)^{2}\right)-\frac{1}{2}\left(C^{\prime}\right)^{2}\right. & \left.-\frac{1}{2}\left(C^{\prime} \cdot K_{X}\right)\right) \\
& =C^{\prime} \cdot C+\frac{v}{2}\left(K_{X} \cdot C^{\prime}\right),
\end{aligned}
$$

so

$$
C^{\prime} \cdot C=(u+\beta v)\left(C^{\prime} \cdot H\right)+v\left(C^{\prime} \cdot D\right)-\frac{v}{2}\left(C^{\prime} \cdot K_{X}\right)
$$

for every class $C^{\prime}$. Thus for any class $C^{\prime}$ with $C^{\prime} \cdot H=0$, we have $C^{\prime} \cdot C=$ $v\left(C^{\prime} \cdot D\right)-\frac{1}{2} v\left(C^{\prime} \cdot K_{X}\right)$; it follows that there is some number $a$ with

$$
C=-\frac{v}{2} K_{X}+a H+v D .
$$

Considering $C=H$ shows that $a=u+\beta v$. Therefore,

$$
\boldsymbol{w}_{\sigma}=\left(-v,-\frac{v}{2} K_{X}+(u+\beta v) H, m\right),
$$

where $m$ is chosen such that $\boldsymbol{w}_{\sigma} \in \boldsymbol{v}^{\perp}$.

Finally, a straightforward calculation shows that

$$
\frac{u}{v}+\beta=v_{\sigma}(v)+\beta=s_{W}
$$

holds for all $(\beta, \alpha)$ along $W$. The follow-up statement for Hilbert schemes follows by computing the Donaldson homomorphism.

3D. Dual curves. Suppose $D_{\sigma_{0}}$ is the nef divisor corresponding to the Gieseker wall for $X^{[n]}$ in the $(H, D)$-slice. Showing that $D_{\sigma_{0}}$ is an extremal nef divisor amounts to showing that there is some curve $\gamma \subset X^{[n]}$ with $D_{\sigma_{0}} \cdot \gamma=0$. By the positivity lemma, this happens when $\gamma$ parametrizes objects of $X^{[n]}$ which are generically $S$-equivalent with respect to $\sigma_{0}$.

In every case where we computed the Gieseker wall, the wall can be given by a destabilizing subobject which is a line bundle $\mathcal{O}_{X}(-C)$ with $C$ an effective curve. If $Z$ is a length- $n$ subscheme of $C$, then there is a destabilizing sequence

$$
0 \rightarrow \mathcal{O}_{X}(-C) \rightarrow I_{Z} \rightarrow I_{Z \subset C} \rightarrow 0 .
$$

If $\operatorname{ext}^{1}\left(I_{Z \subset C}, \mathcal{O}_{X}(-C)\right) \geq 2$, then curves of objects of $X^{[n]}$ which are generically $S$-equivalent with respect to $\sigma_{0}$ are obtained by varying the extension class. We obtain the following general result.

Lemma 3.10. Suppose the Gieseker wall for $X^{[n]}$ in the $(H, D)$-slice is computed by the subobject $\mathcal{O}_{X}(-C)$, where $C$ is an effective curve class of arithmetic genus $p_{a}(C)$. If $n \geq p_{a}(C)+1$, then the corresponding nef divisor $D_{\sigma_{0}}$ is extremal. 
Proof. Bilinearity of the Euler characteristic $\chi(\cdot, \cdot)$ and Serre duality show that

$$
\chi\left(I_{Z \subset C}, \mathcal{O}_{X}(-C)\right)=p_{a}(C)-1-n .
$$

Therefore, once $n \geq p_{a}(C)+1$ we will have $\chi\left(I_{Z \subset C}, \mathcal{O}_{X}(-C)\right) \leq-2$, and curves orthogonal to $D_{\sigma_{0}}$ can be constructed by varying the extension class.

Combining Lemma 3.10 with our previous results on the computation of the Gieseker wall gives us the following asymptotic result.

Theorem 3.11. Fix a slice $(H, D)$ for $\operatorname{Stab}(X)$. There is some $L \in \operatorname{CrDiv}(H, D)$ such that for all $n \gg 0$ the Gieseker wall is computed by $\mathcal{O}_{X}(-L)$. Furthermore, the corresponding nef divisor is extremal.

Proof. Recall that the set $\operatorname{CrDiv}(H, D) / \sim$ of critical divisors modulo numerical equivalence is finite; say $\left\{L_{1}, \ldots, L_{m}\right\}$ is a set of representatives. For $1 \leq i \leq m$, let $\left(s_{i}(n), 0\right)$ be the center of the wall $\mathcal{O}_{X}\left(-L_{i}\right)$ for $X^{[n]}$. Then $s_{i}(n)$ is a linear function of $n$ by Lemma 3.3, so there is some $i$ with $s_{i}(n) \leq s_{j}(n)$ for all $1 \leq j \leq m$ and $n \gg 0$. Then by Corollary 3.7 the Gieseker wall is given by $\mathcal{O}_{X}\left(-L_{i}\right)$. Again increasing $n$ if necessary, the divisor $D_{\sigma_{0}}$ corresponding to the Gieseker wall is extremal by Lemma 3.10 .

Remark 3.12. The requirement $n \geq p_{a}(C)+1$ in Lemma 3.10 is not typically sharp. For example, if $|C|$ contains a smooth curve we may as well assume $C$ is smooth. Then $I_{Z \subset C}$ is a line bundle on $C$, and

$$
\operatorname{Ext}^{1}\left(I_{Z \subset C}, \mathcal{O}_{X}(-C)\right) \cong H^{0}\left(\mathcal{O}_{C}(Z)\right) .
$$

Thus a $g_{n}^{1}$ on $C$ gives a curve which is orthogonal to $D_{\sigma_{0}}$. The following fact from Brill-Noether theory therefore provides curves on $X^{[n]}$ for smaller values on $n$.

Lemma 3.13 [Arbarello et al. 1985]. If $C$ is smooth of genus $g$, then it has a $g_{n}^{1}$ for any $n \geq\lceil(g+2) / 2\rceil$.

For specific surfaces, some curves in $|C|$ may have highly special linear series giving better constructions of curves on $X^{[n]}$.

\section{Picard rank 1 examples}

For the rest of the paper, we will apply the methods of Section 3 to compute $\operatorname{Nef}\left(X^{[n]}\right)$ for several interesting surfaces $X$. These applications form the heart of the paper.

4A. Picard rank 1 in general. Suppose $\operatorname{Pic}(X) \cong \mathbb{Z} H$ for some ample divisor $H$. If we choose $D=-a H$, where $a>0$ is the smallest positive integer such that $a H$ is effective, then $\operatorname{CrDiv}(H, D)=\{-D\}$. 
Lemma 4.1. Suppose $\operatorname{Pic}(X)=\mathbb{Z} H$ and $a H$ is the minimal effective class. If $n \geq(a H)^{2}=a^{2} d$, then the Gieseker wall for $X^{[n]}$ is the wall given by $\mathcal{O}_{X}(-a H)$. Proof. Apply Corollary 3.7 with $D=-a H$.

Note that when $n>a^{2} d$, additional information about the Jordan-Hölder filtration can be obtained as in Corollary 3.7. We use formula (7) to see that the wall $W$ given by $\mathcal{O}_{X}(-a H)$ has center $\left(s_{W}, 0\right)$ with

$$
s_{W}=\frac{a}{2}-\frac{n}{a d} .
$$

Combining Lemmas 4.1 and 3.10 with Proposition 3.8, we have proved the following general result.

Theorem 4.2. Suppose Pic $X \cong \mathbb{Z} H$ and $a H$ is the minimal effective class. If $n \geq a^{2} d$ then the divisor

$$
\frac{1}{2} K_{X}^{[n]}+\left(\frac{a}{2}+\frac{n}{a d}\right) H^{[n]}-\frac{1}{2} B
$$

is nef. Additionally, if $n \geq p_{a}(a H)+1$ then this divisor is extremal, so $\operatorname{Nef}\left(X^{[n]}\right)$ is spanned by this divisor and $H^{[n]}$. An orthogonal curve is given by letting $n$ points move in a $g_{n}^{1}$ on a curve of class $a H$.

Remark 4.3. If $\operatorname{Pic}(X)=\mathbb{Z} H$ and $H$ is already effective, then a different argument computes the Gieseker wall so long as $2 n>d$, improving the bound in Lemma 4.1. However, fine information about the Jordan-Hölder filtration of a destabilized ideal sheaf is not obtained. In fact, if $n \leq d$ then the destabilizing behavior can be complicated. For instance, a scheme $Z$ contained in the complete intersection of two curves of class $H$ will admit an interesting map from $\mathcal{O}_{X}(-H)^{\oplus 2}$.

Proposition 4.4. Suppose Pic $X=\mathbb{Z} H$ and $H$ is effective. If $2 n>d$, then the Gieseker wall for $X^{[n]}$ in the $(H,-H)$-slice is the wall given by $\mathcal{O}_{X}(-H)$. Thus the divisor (8) with $a=1$ is nef.

Proof. Let $W$ be the numerical wall given by $\mathcal{O}_{X}(-H)$. If no actual wall is larger than $W$ then, by the proof of Proposition 3.5, $W$ is an actual wall, and hence the Gieseker wall. If there is a destabilizing sequence

$$
0 \rightarrow F \rightarrow I_{Z} \rightarrow G \rightarrow 0
$$

giving a wall $W^{\prime}$ larger than $W$, then $F, G \in \mathcal{A}_{0}$ since $W$ passes through the origin in the $(\beta, \alpha)$-plane. Fix $\alpha>0$ such that $(0, \alpha)$ lies on $W^{\prime}$. We have

$$
H \cdot \operatorname{ch}_{1}^{-H}(F)=\Im Z_{0, \alpha}(F) \geq 0 \quad \text { and } \quad H \cdot \operatorname{ch}_{1}^{-H}(G)=\Im Z_{0, \alpha}(G) \geq 0 .
$$

Since $d$ is the smallest intersection number of $H$ with an integral divisor and

$$
d=\Im Z_{0, \alpha}\left(I_{Z}\right)=\Im Z_{0, \alpha}(F)+\Im Z_{0, \alpha}(G),
$$


we conclude that either $\Im Z_{0, \alpha}(F)=0$ or $\Im Z_{0, \alpha}(G)=0$. Thus either $F$ or $G$ has infinite $\sigma_{0, \alpha}$-slope, contradicting the fact that $(0, \alpha)$ is on $W^{\prime}$.

We now further relax the lower bound on $n$ needed to guarantee the existence of orthogonal curve classes in special cases.

4B. Surfaces in $\mathbb{P}^{3}$. By the Noether-Lefschetz theorem, a very general surface $X \subset \mathbb{P}^{3}$ of degree $d \geq 4$ is smooth of Picard rank 1 and irregularity 0 . Let $H$ be the hyperplane class and put $D=-H$. We have $K_{X}=(d-4) H$, so Proposition 4.4 shows that if $2 n>d$ then the divisor

$$
\left(\frac{d}{2}-\frac{3}{2}+\frac{n}{d}\right) H^{[n]}-\frac{1}{2} B
$$

is nef. If $C$ is any smooth hyperplane section then the projection from a point on $C$ gives a map of degree $d-1$ to $\mathbb{P}^{1}$, so $C$ carries a $g_{n}^{1}$ for any $n \geq d-1$. We have proved the following result.

Proposition 4.5. Let $X$ be a smooth degree-d hypersurface in $\mathbb{P}^{3}$ with Picard rank 1. The divisor

$$
\left(\frac{d}{2}-\frac{3}{2}+\frac{n}{d}\right) H^{[n]}-\frac{1}{2} B
$$

on $X^{[n]}$ is nef if $2 n>d$. If $n \geq d-1$, then it is extremal, and together with $H^{[n]}$ it spans $\operatorname{Nef}\left(X^{[n]}\right)$.

Remark 4.6. The behavior of $\operatorname{Nef}\left(X^{[n]}\right)$ for smaller $n$ in Proposition 4.5 is more mysterious. Even the cases $d=5$ and $n=2,3$ are interesting.

Remark 4.7. The case $d=4$ of Proposition 4.5 recovers a special case of [Bayer and Macrì 2014b, Proposition 10.3] for K3 surfaces. The case $d=1$ recovers the computation of the nef cone of $\mathbb{P}^{2[n]}$ [Arcara et al. 2013].

4C. Branched covers of $\mathbb{P}^{2}$. Next we consider cyclic branched covers of $\mathbb{P}^{2}$. Let $X$ be a very general cyclic degree- $d$ cover of $\mathbb{P}^{2}$, branched along a degree- $e$ curve. Note that this means that $d$ necessarily divides $e$. We can view these covers as hypersurfaces in a weighted projective space, which gives us a Noether-Lefschetz type theorem: Pic $X=\mathbb{Z} H$, generated by the pullback $H$ of the hyperplane class on $\mathbb{P}^{2}$, provided that $X$ has positive geometric genus. The canonical bundle of $X$ is

$$
K_{X}=-3 H+e\left(\frac{d-1}{d}\right) H=\left(\frac{e(d-1)}{d}-3\right) H .
$$

Then $X$ will have positive geometric genus if $e \geq 3 d /(d-1)$.

Setting $D=-H$, we see that if $2 n>d$ then the divisor class

$$
\left(\frac{e(d-1)}{2 d}-1+\frac{n}{d}\right) H^{[n]}-\frac{1}{2} B
$$


is nef by Proposition 4.4. The preimage of a line is a curve of class $H$, and it carries a $g_{d}^{1}$ given by the map to $\mathbb{P}^{2}$. Therefore, the above divisor is extremal once $n \geq d$. Proposition 4.8. Let $X$ be a very general degree-d cyclic cover of $\mathbb{P}^{2}$ ramified along a degree-e curve, where $d$ divides e and $e \geq 3 d /(d-1)$. The divisor

$$
\left(\frac{e(d-1)}{2 d}-1+\frac{n}{d}\right) H^{[n]}-\frac{1}{2} B
$$

on $X^{[n]}$ is nef if $2 n>d$. For $n \geq d$, this class is extremal, and together with $H^{[n]}$ it spans $\operatorname{Nef}\left(X^{[n]}\right)$.

\section{Del Pezzo surfaces of degree 1}

Bertram and Coskun [2013] studied the birational geometry of $X^{[n]}$ when $X$ is a minimal rational surface or a del Pezzo surface. In particular, they completely computed the nef cones of all these Hilbert schemes except in the case of a del Pezzo surface of degree 1 . The constructions they gave were classical: they produced nef divisors from $k$-very ample line bundles, and dual curves by letting collections of points move in linear pencils on special curves.

In this section, we will compute the nef cone of $X^{[n]}$, where $X$ is a smooth del Pezzo surface of degree 1 . Then $X \cong \mathrm{Bl}_{p_{1}, \ldots, p_{8}} \mathbb{P}^{2}$ for distinct points $p_{1}, \ldots, p_{8}$ with the property that $-K_{X}$ is ample (see [Manin 1974, Theorem 24.4] or [Beauville 1996, Exercise V.21.1]). This application exhibits the full strength of the methods of Section 3.

5A. Notation and statement of results. Let $H$ be the class of a line and let the 8 exceptional divisors over the $p_{i}$ be $E_{1}, \ldots, E_{8}$, so $\operatorname{Pic}(X) \cong \mathbb{Z} H \oplus \mathbb{Z} E_{1} \oplus \cdots \oplus \mathbb{Z} E_{8}$ and $K_{X}=-3 H+\sum_{i} E_{i}$. Recall that a $(-1)$-curve on $X$ is a smooth rational curve of self-intersection -1 . It is simplest to describe the dual cone of effective curves. We recommend reviewing Section 2A for notation.

Theorem 5.1. The cone of curves $\mathrm{NE}\left(X^{[n]}\right)$ is spanned by all the classes $E_{[n]}$ given by (-1)-curves $E \subset X$, the class of a curve contracted by the Hilbert-Chow morphism, and the class $F_{[n]}$, where $F \in\left|-K_{X}\right|$ is an anticanonical curve.

The $240(-1)$-curves $E$ on $X$ are well-known. The possible classes are
$(0 ; 1)$,
$\left(1 ; 1^{2}\right)$,
$\left(2 ; 1^{5}\right)$,
$\left(3 ; 2,1^{6}\right)$,
$\left(4 ; 2^{3}, 1^{5}\right)$,
$\left(5 ; 2^{6}, 1^{2}\right)$,
$\left(6 ; 3,2^{7}\right)$,

where, e.g., $\left(4 ; 2^{3}, 1^{5}\right)$ denotes any class equivalent to

$$
4 H-2 E_{1}-2 E_{2}-2 E_{3}-E_{4}-E_{5}-E_{6}-E_{7}-E_{8}
$$

under the natural action of $S_{8}$ on $\operatorname{Pic}(X)$. The cone of curves $\mathrm{NE}(X)$ is spanned by the classes of the $(-1)$-curves. The Weyl group action on $\operatorname{Pic}(X)$ acts transitively 
on (-1)-curve classes. It also acts transitively on systems of 8 pairwise disjoint $(-1)$-curves; dually, it acts transitively on the extremal rays of the nef cone $\operatorname{Nef}(X)$. We refer the reader to [Manin 1974, §26] for details.

Consider the divisor class $(n-1)\left(-K_{X}\right)^{[n]}-\frac{1}{2} B$. If $E$ is any $(-1)$-curve on $X$, then $-K_{X} \cdot E=1$, so

$$
E_{[n]} \cdot\left((n-1)\left(-K_{X}\right)^{[n]}-\frac{1}{2} B\right)=(n-1)\left(-K_{X} \cdot E\right)-(n-1)=0 .
$$

Let $\Lambda \subset N^{1}\left(X^{[n]}\right)$ be the cone spanned by divisors which are nonnegative on all classes $E_{[n]}$ and curves contracted by the Hilbert-Chow morphism. It follows that $\Lambda \supset \operatorname{Nef}\left(X^{[n]}\right)$ is spanned by $\operatorname{Nef}(X) \subset \operatorname{Nef}\left(X^{[n]}\right)$ and the single additional class $(n-1)\left(-K_{X}\right)^{[n]}-\frac{1}{2} B$.

However, $\operatorname{Nef}\left(X^{[n]}\right) \subset \Lambda$ is a proper subcone. Indeed, if $F \in\left|-K_{X}\right|$ is an anticanonical curve then by Riemann-Hurwitz $F_{[n]} \cdot B=2 n$, so $F_{[n]} \cdot\left((n-1)\left(-K_{X}\right)^{[n]}-\right.$ $\left.\frac{1}{2} B\right)=-1$. Let $\Lambda^{\prime} \subset \Lambda$ be the subcone of $F_{[n]}$-nonnegative divisors. Taking duals, we see that Theorem 5.1 is equivalent to the next result.

Theorem 5.2. The nef cone of $X^{[n]}$ is $\Lambda^{\prime}$.

To prove Theorem 5.2, we must show that all the extremal rays of $\Lambda^{\prime}$ are actually nef. Suppose $N \in \operatorname{Nef}(X)$ spans an extremal ray of $\operatorname{Nef}(X)$. Then the cone spanned by $N^{[n]}$ and $(n-1)\left(-K_{X}\right)^{[n]}-\frac{1}{2} B$ contains a single ray of $F_{[n]}$-orthogonal divisors, and this ray is an extremal ray of $\Lambda^{\prime}$. Conversely, due to our description of the cone $\Lambda$, the extremal rays of $\Lambda^{\prime}$ which are not in $\operatorname{Nef}(X)$ are all obtained in this way.

5B. Choosing a slice. More concretely, making use of the Weyl group action we may as well assume our extremal nef class $N \in \operatorname{Nef}(X)$ is $H-E_{1}$. The corresponding $F_{[n]}$-orthogonal ray described in the previous paragraph is spanned by

$$
(n-1)\left(-K_{X}\right)^{[n]}+\frac{1}{2}\left(H^{[n]}-E_{1}^{[n]}\right)-\frac{1}{2} B
$$

our job is to show that this class is nef. We will prove this by exhibiting this divisor as the nef divisor on $X^{[n]}$ corresponding to the Gieseker wall for a suitable choice of slice of $\operatorname{Stab}(X)$.

To apply the methods of Section 3, it is convenient to choose our polarization to be

$$
P=\left(n-\frac{3}{2}\right)\left(-K_{X}\right)+\frac{1}{2}\left(H-E_{1}\right)
$$

(which depends on $n !$ ) and our antieffective class to be $D=K_{X}$. Observe that $P$ is ample since it is the sum of an ample and a nef class. If we show that the Gieseker wall $W$ in the $\left(P, K_{X}\right)$-slice has center $\left(s_{W}, 0\right)=(-1,0)$, then Proposition 3.8 implies the divisor class (9) is nef. 
5C. Critical divisors. Our plan is to apply Corollary 3.7 to compute the Gieseker wall in the $\left(P, K_{X}\right)$-slice. We must first identify the set $\operatorname{CrDiv}\left(P, K_{X}\right)$ of critical divisors.

Lemma 5.3. If $n>2$, then the set $\operatorname{CrDiv}\left(P, K_{X}\right)$ consists of $-K_{X}$ and the classes $L$ of $(-1)$-curves on $X$ with $L \cdot\left(H-E_{1}\right) \leq 1$.

When $n=2$, the above classes are still critical. Additionally, the class $H-E_{1}$ is critical, as is any sum of two $(-1)$-curves $L_{1}, L_{2}$ with $L_{i} \cdot\left(H-E_{1}\right)=0$.

Proof. Write $2 P=A+N$, where $A=(2 n-3)\left(-K_{X}\right)$ is ample and $N=H-E_{1}$ is nef. Then $A \cdot\left(-K_{X}\right)=2 n-3$ and $N \cdot\left(-K_{X}\right)=2$, so an effective curve class $L \neq-K_{X}$ is in $\operatorname{CrDiv}\left(P, K_{X}\right)$ if and only if $L \cdot(2 P)<2 n-1$.

First suppose $n>2$, and let $L \in \operatorname{CrDiv}\left(P, K_{X}\right)$. If $L \cdot\left(-K_{X}\right) \geq 2$, then $L \cdot(2 P) \geq$ $4 n-6>2 n-1$, so $L$ is not critical. Therefore, $L \cdot\left(-K_{X}\right)=1$. Thus any curve of class $L$ is reduced and irreducible. By the Hodge index theorem,

$$
L^{2}=L^{2} \cdot\left(-K_{X}\right)^{2} \leq\left(L \cdot\left(-K_{X}\right)\right)^{2}=1,
$$

with equality if and only if $L=-K_{X}$. If the inequality is strict, then by adjunction we must have $L^{2}=-1$ and $L$ is a $(-1)$-curve. Since $L \cdot(2 P)<2 n-1$, we further have $L \cdot N \leq 1$.

Suppose instead that $n=2$ and $L \in \operatorname{CrDiv}\left(P, K_{X}\right)$. The cases $L \cdot(2 P) \leq 1$ and $L \cdot(2 P) \geq 3$ follow as in the previous case. The only other possibility is that $L \cdot\left(-K_{X}\right)=2$ and $L \cdot N=0$. Since $L \cdot N=0$, the curve $L$ is a sum of curves in fibers of the projection $X \rightarrow \mathbb{P}^{1}$ given by $|N|$. This easily implies the result.

The next application of Corollary 3.7 completes the proof of Theorems 5.1 and 5.2.

Proposition 5.4. The Gieseker wall for $X^{[n]}$ in the $\left(P, K_{X}\right)$-slice has center $(-1,0)$, and is given by the subobject $\mathcal{O}_{X}\left(K_{X}\right)$. It coincides with the wall given by $\mathcal{O}_{X}(-L)$, where $L$ is any $(-1)$-curve with $L \cdot\left(H-E_{1}\right)=0$.

Proof. By (7), the center of the wall for $\mathcal{O}_{X}\left(K_{X}\right)$ is $\left(s_{W}, 0\right)$ with

$$
s_{W}=\frac{2 n-K_{X}^{2}}{(2 P) \cdot K_{X}}=-1 .
$$

A straightforward computation shows $\eta_{P, K_{X}}<n$ for all $n \geq 2$. Therefore, by Corollary 3.7, the Gieseker wall is computed by a critical divisor.

We only need to verify that no other critical divisor gives a larger wall. Let $L \in \operatorname{CrDiv}\left(P, K_{X}\right)$. By Lemma 3.3, the center of the wall given by $\mathcal{O}_{X}(-L)$ lies at the point $\left(s_{L}, 0\right)$, where

$$
s_{L}=-\frac{2 n+L^{2}+2\left(K_{X} \cdot L\right)}{(2 P) \cdot L} .
$$


If $L$ is a $(-1)$-curve, then

$$
s_{L}=-\frac{2 n-3}{(2 P) \cdot L}=-\frac{2 n-3}{2 n-3+L \cdot\left(H-E_{1}\right)} \geq-1,
$$

with equality if and only if $L \cdot\left(H-E_{1}\right)=0$. This proves the result if $n>2$.

To complete the proof when $n=2$, we only need to consider the additional critical classes mentioned in Lemma 5.3. For every such $L \in \operatorname{CrDiv}\left(P, K_{X}\right)$ we have $L \cdot K_{X}=-2$ and $L^{2} \leq 0$. Thus $s_{L} \geq 0$ for every such divisor.

\section{Acknowledgements}

This work was initiated at the 2015 Algebraic Geometry Bootcamp preceding the Algebraic Geometry Summer Research Institute organized by the AMS and the University of Utah. We would like to thank the organizers of both programs for providing the wonderful environment where this collaboration could happen. Additionally, we would like to thank Arend Bayer, Izzet Coskun, and Emanuele Macrì for many valuable discussions on Bridgeland stability.

\section{References}

[Arbarello et al. 1985] E. Arbarello, M. Cornalba, P. A. Griffiths, and J. Harris, Geometry of algebraic curves, vol. I, Grundlehren der Math. Wissenschaften 267, Springer, New York, 1985. MR 770932 Zbl 0559.14017

[Arcara and Bertram 2013] D. Arcara and A. Bertram, "Bridgeland-stable moduli spaces for $K$-trivial surfaces”, J. Eur. Math. Soc. (JEMS) 15:1 (2013), 1-38. MR 2998828 Zbl 1259.14014

[Arcara et al. 2013] D. Arcara, A. Bertram, I. Coskun, and J. Huizenga, "The minimal model program for the Hilbert scheme of points on $\mathbb{P}^{2}$ and Bridgeland stability”, Adv. Math. 235 (2013), 580-626. MR 3010070 Zbl 1267.14023

[Bayer and Macrì 2014a] A. Bayer and E. Macrì, "MMP for moduli of sheaves on K3s via wallcrossing: nef and movable cones, Lagrangian fibrations", Invent. Math. 198:3 (2014), 505-590. MR 3279532 Zbl 1308.14011

[Bayer and Macrì 2014b] A. Bayer and E. Macrì, "Projectivity and birational geometry of Bridgeland moduli spaces", J. Amer. Math. Soc. 27:3 (2014), 707-752. MR 3194493 Zbl 1314.14020

[Bayer et al. 2014] A. Bayer, E. Macrì, and P. Stellari, "The Space of Stability Conditions on Abelian Threefolds, and on some Calabi-Yau Threefolds", preprint, 2014. arXiv 1410.1585

[Beauville 1996] A. Beauville, Complex algebraic surfaces, 2nd ed., London Mathematical Society Student Texts 34, Cambridge University Press, 1996. MR 1406314 Zbl 0849.14014

[Beltrametti and Sommese 1991] M. Beltrametti and A. J. Sommese, "Zero cycles and $k$-th order embeddings of smooth projective surfaces", pp. 33-48 in Problems in the theory of surfaces and their classification (Cortona, 1988), edited by F. Catanese et al., Sympos. Math. XXXII, Academic Press, London, 1991. MR 1273371 Zbl 0827.14029

[Beltrametti et al. 1989] M. Beltrametti, P. Francia, and A. J. Sommese, "On Reider's method and higher order embeddings", Duke Math. J. 58:2 (1989), 425-439. MR 1016428 Zbl 0702.14010 
[Bertram and Coskun 2013] A. Bertram and I. Coskun, "The birational geometry of the Hilbert scheme of points on surfaces", pp. 15-55 in Birational geometry, rational curves, and arithmetic, edited by F. Bogomolov et al., Springer, New York, 2013. MR 3114922 Zbl 1273.14032

[Bertram et al. 2014] A. Bertram, C. Martinez, and J. Wang, "The birational geometry of moduli spaces of sheaves on the projective plane", Geom. Dedicata 173 (2014), 37-64. MR 3275289 Zbl 1314.14087

[Bridgeland 2007] T. Bridgeland, "Stability conditions on triangulated categories", Ann. of Math. (2) 166:2 (2007), 317-345. MR 2373143 Zbl 1137.18008

[Bridgeland 2008] T. Bridgeland, "Stability conditions on $K 3$ surfaces", Duke Math. J. 141:2 (2008), 241-291. MR 2376815 Zbl 1138.14022

[Catanese and Göttsche 1990] F. Catanese and L. Göttsche, " $d$-very-ample line bundles and embeddings of Hilbert schemes of 0-cycles”, Manuscripta Math. 68:3 (1990), 337-341. MR 1065935 Zbl 0729.14006

[Coskun and Huizenga 2014] I. Coskun and J. Huizenga, "Interpolation, Bridgeland stability and monomial schemes in the plane", J. Math. Pures Appl. (9) 102:5 (2014), 930-971. MR 3271294 Zbl 06361756

[Coskun and Huizenga 2016] I. Coskun and J. Huizenga, "The ample cone of moduli spaces of sheaves on the plane", Algebr. Geom. 3:1 (2016), 106-136. MR 3455422

[Ellingsrud et al. 2001] G. Ellingsrud, L. Göttsche, and M. Lehn, "On the cobordism class of the Hilbert scheme of a surface”, J. Algebraic Geom. 10:1 (2001), 81-100. MR 1795551 Zbl 0976.14002

[Fogarty 1968] J. Fogarty, "Algebraic families on an algebraic surface", Amer. J. Math 90 (1968), 511-521. MR 0237496 Zbl 0176.18401

[Fogarty 1973] J. Fogarty, "Algebraic families on an algebraic surface, II: The Picard scheme of the punctual Hilbert scheme", Amer. J. Math. 95 (1973), 660-687. MR 0335512 Zbl 0299.14020

[Hartshorne 1977] R. Hartshorne, Algebraic geometry, Graduate Texts in Mathematics 52, Springer, New York, 1977. MR 0463157 Zbl 0367.14001

[Hassett and Tschinkel 2010] B. Hassett and Y. Tschinkel, "Intersection numbers of extremal rays on holomorphic symplectic varieties", Asian J. Math. 14:3 (2010), 303-322. MR 2755719 Zbl 1216.14012

[Lazarsfeld 2004] R. Lazarsfeld, Positivity in algebraic geometry, I: Classical setting: line bundles and linear series, Ergebnisse der Mathematik (3) 48, Springer, Berlin, 2004. MR 2095471 Zbl 1093.14501

[Li and Zhao 2013] C. Li and X. Zhao, "The MMP for deformations of Hilbert schemes of points on the projective plane", preprint, 2013. arXiv 1312.1748

[Liu 2015] W. Liu, "Bayer-Macrì decomposition on Bridgeland moduli spaces over surfaces", preprint, 2015. arXiv 1501.06397

[Maciocia 2014] A. Maciocia, "Computing the walls associated to Bridgeland stability conditions on projective surfaces”, Asian J. Math. 18:2 (2014), 263-279. MR 3217637 Zbl 1307.14022

[Maciocia and Meachan 2013] A. Maciocia and C. Meachan, "Rank 1 Bridgeland stable moduli spaces on a principally polarized abelian surface", Int. Math. Res. Not. 2013:9 (2013), 2054-2077. MR 3053413 Zbl 1312.14036

[Manin 1974] Y. I. Manin, Cubic forms: algebra, geometry, arithmetic, North-Holland, Amsterdam, 1974. MR 0460349 Zbl 0277.14014

[Matsuki and Wentworth 1997] K. Matsuki and R. Wentworth, "Mumford-Thaddeus principle on the moduli space of vector bundles on an algebraic surface", Internat. J. Math. 8:1 (1997), 97-148. MR 1433203 Zbl 0879.14002 
930 B. Bolognese, J. Huizenga, Y. Lin, E. Riedl, B. Schmidt, M. Woolf and X. Zhao

[Nuer 2014] H. Nuer, "Projectivity and Birational Geometry of Bridgeland Moduli spaces on an Enriques Surface", preprint, 2014. arXiv 1406.0908

[Yanagida and Yoshioka 2014] S. Yanagida and K. Yoshioka, "Bridgeland's stabilities on abelian surfaces”, Math. Z. 276:1-2 (2014), 571-610. MR 3150219 Zbl 1292.14012

Communicated by János Kollár

Received 2015-11-01 Revised 2016-03-12 Accepted 2016-05-07

bolognese.b@husky.neu.edu Department of Mathematics, Northeastern University, Boston, MA 02115, United States

huizenga@psu.edu

Department of Mathematics, The Pennsylvania State University, University Park, PA 16802, United States

lin.yinb@husky.neu.edu

Department of Mathematics, Northeastern University, Boston, MA 02115, United States

ebriedI@uic.edu

Department of Mathematics, Statistics, and CS, University of Illinois, Chicago, IL 60607, United States

schmidt.707@osu.edu

Department of Mathematics, The Ohio State University, Columbus, $\mathrm{OH}$ 43210, United States

mwoolf@ed.ac.uk

Department of Mathematics, Statistics, and CS, University of Illinois, Chicago, IL 60607, United States

x.zhao@neu.edu

Department of Mathematics, Northeastern University, Boston, MA 02115, United States 


\section{Algebra \& Number Theory}

msp.org/ant

\section{EDITORS}

MANAGING EDITOR

Bjorn Poonen

Massachusetts Institute of Technology

Cambridge, USA

\author{
EDITORIAL BOARD CHAIR \\ David Eisenbud \\ University of California \\ Berkeley, USA
}

BOARD OF EDITORS

Georgia Benkart

Dave Benson

Richard E. Borcherds

John H. Coates

J-L. Colliot-Thélène

Brian D. Conrad

Hélène Esnault

Hubert Flenner

Sergey Fomin

Edward Frenkel

Andrew Granville

Joseph Gubeladze

Roger Heath-Brown

Craig Huneke

Kiran S. Kedlaya

János Kollár

Yuri Manin

Philippe Michel
University of Wisconsin, Madison, USA

University of Aberdeen, Scotland

University of California, Berkeley, USA

University of Cambridge, UK

CNRS, Université Paris-Sud, France

Stanford University, USA

Freie Universität Berlin, Germany

Ruhr-Universität, Germany

University of Michigan, USA

University of California, Berkeley, USA

Université de Montréal, Canada

San Francisco State University, USA

Oxford University, UK

University of Virginia, USA

Univ. of California, San Diego, USA

Princeton University, USA

Northwestern University, USA

École Polytechnique Fédérale de Lausanne
Susan Montgomery

Shigefumi Mori

Raman Parimala

Jonathan Pila

Anand Pillay

Victor Reiner

Peter Sarnak

Joseph H. Silverman

Michael Singer

Vasudevan Srinivas

J. Toby Stafford

Ravi Vakil

Michel van den Bergh

Marie-France Vignéras

Kei-Ichi Watanabe

Efim Zelmanov

Shou-Wu Zhang
University of Southern California, USA

RIMS, Kyoto University, Japan

Emory University, USA

University of Oxford, UK

University of Notre Dame, USA

University of Minnesota, USA

Princeton University, USA

Brown University, USA

North Carolina State University, USA

Tata Inst. of Fund. Research, India

University of Michigan, USA

Stanford University, USA

Hasselt University, Belgium

Université Paris VII, France

Nihon University, Japan

University of California, San Diego, USA

Princeton University, USA

\section{PRODUCTION}

production@msp.org

Silvio Levy, Scientific Editor

See inside back cover or msp.org/ant for submission instructions.

The subscription price for 2016 is US $\$ 290 /$ year for the electronic version, and $\$ 485 /$ year ( $+\$ 55$, if shipping outside the US) for print and electronic. Subscriptions, requests for back issues and changes of subscribers address should be sent to MSP.

Algebra \& Number Theory (ISSN 1944-7833 electronic, 1937-0652 printed) at Mathematical Sciences Publishers, 798 Evans Hall \#3840, c/o University of California, Berkeley, CA 94720-3840 is published continuously online. Periodical rate postage paid at Berkeley, CA 94704, and additional mailing offices.

ANT peer review and production are managed by EditFLOW ${ }^{\circledR}$ from MSP.

\section{PUBLISHED BY}

- mathematical sciences publishers

nonprofit scientific publishing

http://msp.org/

(C) 2016 Mathematical Sciences Publishers 


\section{Algebra \& Number Theory}

Volume $10 \quad$ No. $4 \quad 2016$

Moduli of morphisms of logarithmic schemes

JONATHAN WISE

Residual intersections and the annihilator of Koszul homologies

SEYED HAMID HaSSANZADEH and Jose NAÉLITON

The Prym map of degree-7 cyclic coverings

HERBERT LANGE and ANGELA ORTEGA

SIMON MARSHALL

Hasse principle for Kummer varieties

Yonatan HaRPaZ and AlEXEI N. SkoRobogatov

Analytic continuation on Shimura varieties with $\mu$-ordinary locus

STÉPHANE BIJAKOWSKI

A note on secondary $K$-theory

GONÇALO TABUADA

Nef cones of Hilbert schemes of points on surfaces

Barbara Bolognese, Jack Huizenga, Yinbang Lin, Eric Riedl,

BENJAMIN SCHMidT, MATTHEW WoOlF and XiaOlei ZHAO

Interpolation for restricted tangent bundles of general curves 